\title{
An integrated analysis of the rice transcriptome and lipidome reveals lipid metabolism plays a central role in rice cold tolerance
}

\author{
Hualong Liu ${ }^{1 \dagger}$, Wei Xin ${ }^{1 \dagger}$, Yinglin Wang ${ }^{1}$, Dezhuang Zhang ${ }^{1}$, Jingguo Wang ${ }^{1}$, Hongliang Zheng ${ }^{1}$,
} Luomiao Yang ${ }^{1}$, Shoujun $\mathrm{Nie}^{2^{*}}$ and Detang Zou ${ }^{1^{*}}$

\begin{abstract}
Background: Rice (Oryza sativa L.) is one of the most widely grown food crops, and its yield and quality are particularly important for a warm-saturated diet. Cold stress restricts rice growth, development, and yield; however, the specific mechanism of cold tolerance in rice remains unknown.

Results: The analysis of leaf physiological and photosynthetic characteristics showed that the two rice varieties were significantly affected by cold stress, but the cold-tolerant variety KY131 had more stable physiological characteristics, maintaining relatively good photosynthetic capacity. To better explore the transcriptional regulation mechanism and biological basis of rice response to cold stress, a comprehensive analysis of the rice transcriptome and lipidome under low temperature and control temperature conditions was carried out. The transcriptomic analysis revealed that lipid metabolism, including membrane lipid and fatty acid metabolism, may be an important factor in rice cold tolerance, and 397 lipid metabolism related genes have been identified. Lipidomics data confirmed the importance of membrane lipid remodeling and fatty acid unsaturation for rice adaptation to cold stress. This indicates that the changes in the fluidity and integrity of the photosynthetic membrane under cold stress lead to the reduction of photosynthetic capacity, which could be relieved by increased levels of monogalactosyldiacylglycerol that mainly caused by markedly increased expression of levels of 1,2-diacylglycerol 3-beta-galactosyltransferase (MGD). The upregulation of phosphatidate phosphatase (PAP2) inhibited the excessive accumulation of phosphatidate (PA) to produce more phosphatidylcholine (PC), phosphatidylethanolamine (PE), and phosphatidylglycerol (PG), thereby preventing of membrane phase transition under cold stress. In addition, fatty acid $\beta$-oxidation is worth further study in rice cold tolerance. Finally, we constructed a metabolic model for the regulatory mechanism of cold tolerance in rice, in which the advanced lipid metabolism system plays a central role.
\end{abstract}

\footnotetext{
*Correspondence: zoudtneau@126.com; nsj-0821@163.com ${ }^{\dagger}$ Hualong Liu and Wei Xin contributed equally to this work.

${ }^{1}$ Key Laboratory of Germplasm Enhancement, Physiology and Ecology

of Food Crops in Cold Region, Ministry of Education, Northeast Agricultural University, 150030 Harbin, China

${ }^{2}$ Innovation Center, Suihua Branch of Heilongjiang Academy

of Agricultural Science, 152052 Suihua, China
}

(C) The Author(s) 2022. Open Access This article is licensed under a Creative Commons Attribution 4.0 International License, which permits use, sharing, adaptation, distribution and reproduction in any medium or format, as long as you give appropriate credit to the original author(s) and the source, provide a link to the Creative Commons licence, and indicate if changes were made. The images or other third party material in this article are included in the article's Creative Commons licence, unless indicated otherwise in a credit line to the material. If material is not included in the article's Creative Commons licence and your intended use is not permitted by statutory regulation or exceeds the permitted use, you will need to obtain permission directly from the copyright holder. To view a copy of this licence, visit http://creativecommons.org/licenses/by/4.0/. The Creative Commons Public Domain Dedication waiver (http://creativeco mmons.org/publicdomain/zero/1.0/) applies to the data made available in this article, unless otherwise stated in a credit line to the data. 
Conclusions: Lipidome analysis showed that membrane lipid composition and unsaturation were significantly affected, especially phospholipids and galactolipids. Our study provides new information to further understand the response of rice to cold stress.

Keywords: Rice, Transcriptome, Lipidomic, Cold stress

\section{Background}

With population growth, the demand for food is increasing daily. Rice (Oryza sativa L.) is one of the most widely grown food crops, and its yield and quality are particularly important for a warm-saturated diet [1]. The rice planting area in the world is approximately 140 million hectares, in particular, more than 15 million hectares are threatened by different degrees of low-temperature damage every year. Chilling damage to rice has led to a reduction in yield and quality, which greatly affects food security, and owing to latitude, this problem is more prominent in China [2,3]. The phenomenon of "spring cold" before and after transplanting rice occurs occasionally. It may delay the growth process of rice, delay the rice plant turning green, reduce the number of tillers, and seriously cause the death of rice seedlings to varying degrees. Some rice varieties with poor cold tolerance die completely, and farmers will need to replant the seedlings. Therefore, cold tolerance of rice germplasm is an unavoidable challenge, for which research can have an important scientific significance in improving cold tolerance of rice and considerably improving the economic value of people's quality of life.

Presently, it is widely considered that the model of plant cold tolerance signal transduction mechanism is initiated when cold stress is first detected by plasma membrane proteins (such as calcium channels or related proteins), leading to calcium influx and then to changes in membrane fluidity and cytoskeletal rearrangement. Then, calcium-responsive protein kinases, including calcium-dependent protein kinases (CDPKs), calcineurin B-like interacting protein kinases (CIPKs), and calciumregulated receptor-like kinases (CRLKs), may mediate calcium signals to activate the mitogen-activated protein kinase (MAPK) cascade, which can regulate cold tolerance-related gene expression and improve cold stress tolerance [4-6]. The expression of $Z m M K K 1$ in maize is induced by various stresses and exogenous signaling molecules. During cold stress, the content of osmotic regulators, antioxidant enzyme activities, and expression of stress-related genes in tobacco plants overexpressing $Z m M K K 1$ are significantly increased, thereby increasing the cold tolerance of genetically modified tobacco [7]. Concurrently, these transcriptome changes are regulated by a complex network of transcription factors (TFs) and other regulatory proteins and RNAs. TFs act as transcriptional activators or repressors and play a central role in the regulation of development, metabolic processes, and biotic and abiotic stresses. Several studies have shown that transcription factors also play an important role in the adaptation of rice to low-temperature stress [8-11].

Previous studies have shown that the regulation mechanism of biofilm fluidity is one of the main mechanisms by which plants adapt to temperature changes, which is affected by the distribution ratio of various lipids on the membrane and the degree of unsaturation [12]. One of the newest research discoveries in the field of cold signal transduction is the formation of lipid second messengers, phosphatidic acid (PA), which accumulates in cells in suspension culture within minutes under cold stress $[13,14]$. During cold-induced signaling, PA responds to cold stress mainly through two pathways: one is a direct product of phospholipase D (PLD), which hydrolyzes structural phospholipids such as phosphatidylcholine (PC) and phosphatidylethanolamine (PE), and the other is a secondary product of the phospholipase C (PLC) pathway, which first polyphosphoinositide (PPI) is hydrolyzed to diacylglycerol (DG), which is subsequently phosphorylated to PA with diacylglycerol kinase (DGK) [15, 16]. Furthermore, PA is the synthetic precursor to all phosphoglycerolipids, turnoveris crucialin, galactolipids, and triacylglycerols (TGs), which determines lipid metabolic flux and membrane composition [17]. Membrane lipid remodeling is another important factor in plant cold tolerance, which has been demonstrated in several algaes, Arabidopsis, and several crops, with specific variations among different plants [18-20]. Previous studies in non-plant systems have shown that in addition to PA, cold stress also induces responses to various lipid signals, such as phosphatidylinositol (PI), lysophospholipids, and sphingolipids [21]. However, how these specific lipid species are produced and further affect plant cold tolerance under cold stress is unclear.

Biomembranes are the barrier for cells and organelles to contact the outside environment and are also the most sensitive to temperature changes. Under low temperature stress, the increase in membrane permeability and decrease in fluidity caused by the phase transition of membrane lipids are the root causes of cold damage to plants [22]. To resist low-temperature damage, plants have formed a complex mechanism 
that adapts to temperature changes through membrane lipid remodeling and adjustment of fatty acid unsaturation during long-term evolution [12, 18, 20]. In this study, cold-tolerant rice varieties Kongyu 131 (KY 131) and cold-sensitive rice varieties Dongnong 422 (DN 422) were used as experimental materials. Using lipid detection technology, the membrane lipid composition and fatty acid (FA) content in the leaves of rice seedlings were determined under low-temperature stress. This was combined with transcriptome data to analyze the lipid metabolism pathway. Clarifying the regulation mode of lipid metabolism in rice under low temperature stress, will establish the basis for analyzing the regulation mechanism of lipid metabolism and improve rice cold tolerance.

\section{Results}

Responses of physiological and photosynthetic characteristics of rice during cold stress

After nine days of cold treatment (Fig. 1 A, B), the two varieties showed withered leaf tips and curled leaves. After five days of recovery treatment (Fig. 1 C), KY131 resumed normal growth except for withered yellow leaf tips, while DN422 showed dry leaves. Based on the cold response of the two varieties, we selected samples processed for nine days to study the relevant physiological and molecular mechanisms. Under cold conditions, the leaf water content in KY 131 and DN 422 decreased, while the water content in KY 131 was significantly higher than that in 422 under cold conditions. The MDA content in KY 131 and DN 422 increased by $130.48 \%$ and $172.40 \%$,
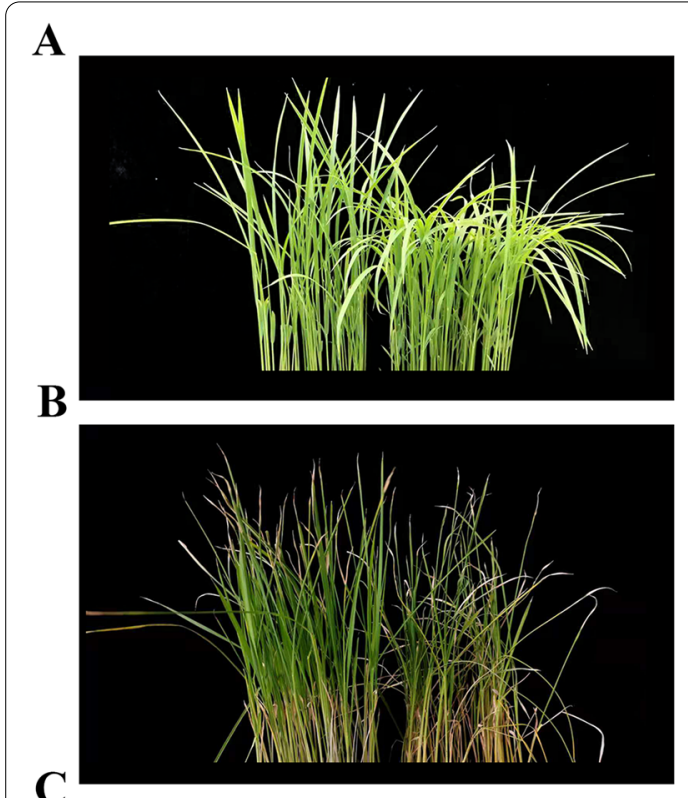

C

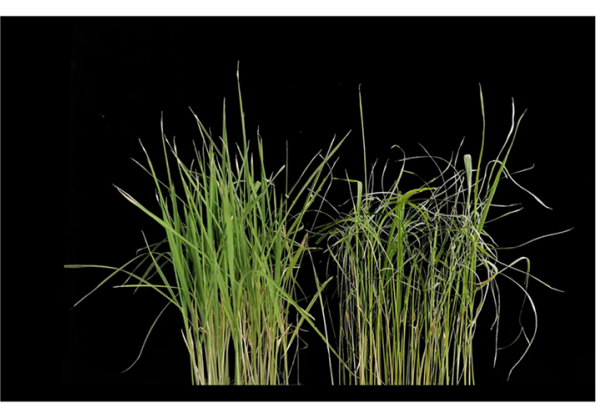

D
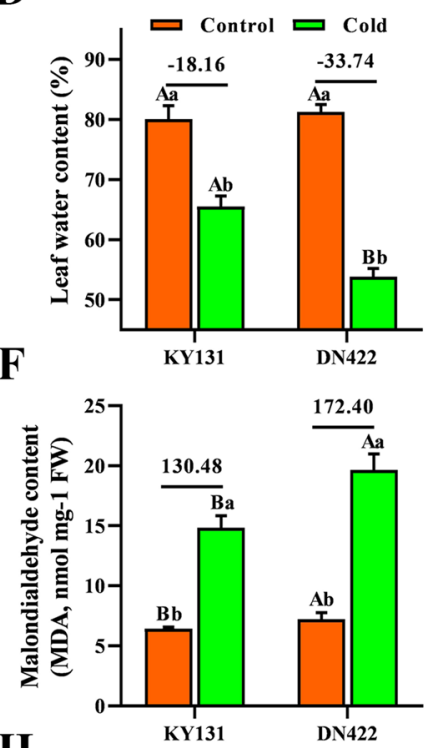

$\mathbf{H}$

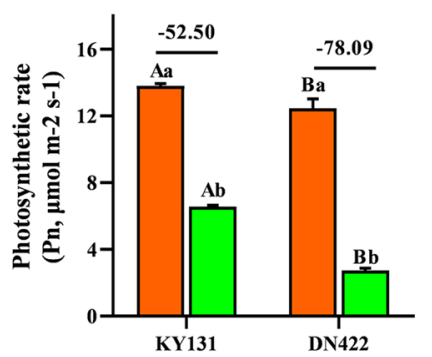

$\mathbf{E}$
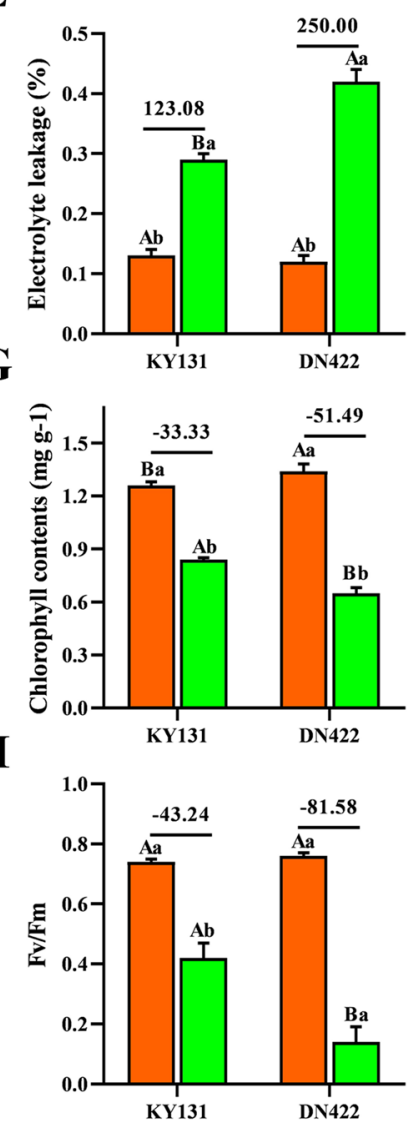

Fig. 1 Leaf phenotypes, physiological and photosynthetic characteristics responses of rice during cold stress. A phenotypes of KY 131 and DN 422 under control conditions; B phenotypes of KY 131 and DN 422 under cold conditions; $\mathbf{C}$ phenotypes of KY 131 and DN 422 under recovery conditions; D leaf water content of KY 131 and DN 422 under cold stress; E electrolyte leakage of KY 131 and DN 422 under cold stress; F malondialdehyde of KY 131 and DN 422 under cold stress; $\mathbf{G}$ the chlorophyll contents of KY 131 and DN 422 under cold stress; $\mathbf{H}$ photosynthetic rate of KY 131 and DN 422 under cold stress; I the maximum photochemical efficiency of PSII of KY 131 and DN 422 under cold stress. Different lowercase and uppercase letters represent significant differences among treatments and cultivars, respectively. (as evaluated with ANOVA with Fisher's LSD, $p<0.05 . n=5$ ) 
respectively. The EL in KY 131 and DN 422 increased by $123.08 \%$ and $250.00 \%$, respectively. The photosynthetic capacity of KY 131 and DN 422 seedlings after nine days of cold stress was estimated by the Chl content, net photosynthetic rate $(\mathrm{Pn})$, and $\mathrm{Fv} / \mathrm{Fm}$, respectively. Under cold conditions, the Chl content, $\mathrm{Pn}$, and $\mathrm{Fv} / \mathrm{Fm}$ were both decreased in the two rice cultivars, while the levels in KY 131 were significantly higher than those in DN 422 (Fig. 1 D-I). These physiological responses indicated that KY 131 was relatively stable under cold conditions.

\section{Cold response transcriptome differences in rice cultivars}

There were significant differences in the physiological and photosynthetic characteristics between KY 131 and DN 422. To understand the regulatory mechanisms of cold stress and explore new regulators, cold-responsive transcriptome changes in the leaves of KY 131 and DN 422 were studied using RNA-seq. Differentially expressed genes (DEGs) were examined using the $R$ package DEseq2 to quantify and analyze all the control and treatment conditions. Over the nine days of cold exposure, 15,986 genes were significantly differentially expressed under cold stress, and of these, 11,394 genes were commonly differentially expressed in both varieties, while 2505 and 2087 genes were specifically expressed in KY131 and DN422, respectively (Tables S1, 2). Moreover, the differential expression level of 1812 genes of 11,394 common differentially expressed genes was significantly higher in KY131 than in DN422 (FC(KY131/DN422) $\geq$
1.5), which comprised the "1812 genes of 11394" and with the 2505 differentially expressed genes only in KY131 and may play a central role in rice cold tolerance (Fig. 2).

\section{The Gene Ontology (GO) and Kyoto Encyclopedia of Genes and Genomes (KEGG) enrichment analysis}

To further determine the functions of the DEGs and the related biological processes they participate in, $\mathrm{GO}$ and KEGG enrichment analyses were conducted. The GO database enrichment analysis was separated into three ontologies: 'cellular component,' 'molecular function,' and 'biological process'. A total of 51 and 53 GO terms were significantly different in the CKKY vs. C9KY and CKDN vs. C9DN comparisons, respectively (Tables S3, 4). We selected the $50 \mathrm{GO}$ terms with the lowest false discovery rate (FDR) in the enrichment analysis results and drew a column chart of enrichment items, as shown in Fig. 3. The results of GO enrichment hierarchical analysis revealed that within the cellular component the enriched GO terms in both KY 131 and DN 422 were 'thylakoid part', 'photosynthetic membrane', 'thylakoid membrane', 'chloroplast thylakoid', and 'plastid thylakoid'. Within the molecular function the enriched GO terms in both $\mathrm{KY}$ 131 and DN 422 were 'chlorophyll binding', 'drug transmembrane transporter activity', 'signaling receptor activity', and 'molecular transducer activity', and the specific term enrichment for KY 131 included 'transmembrane signaling receptor activity'. Within the biological process the enriched GO terms in both KY 131 and DN 422

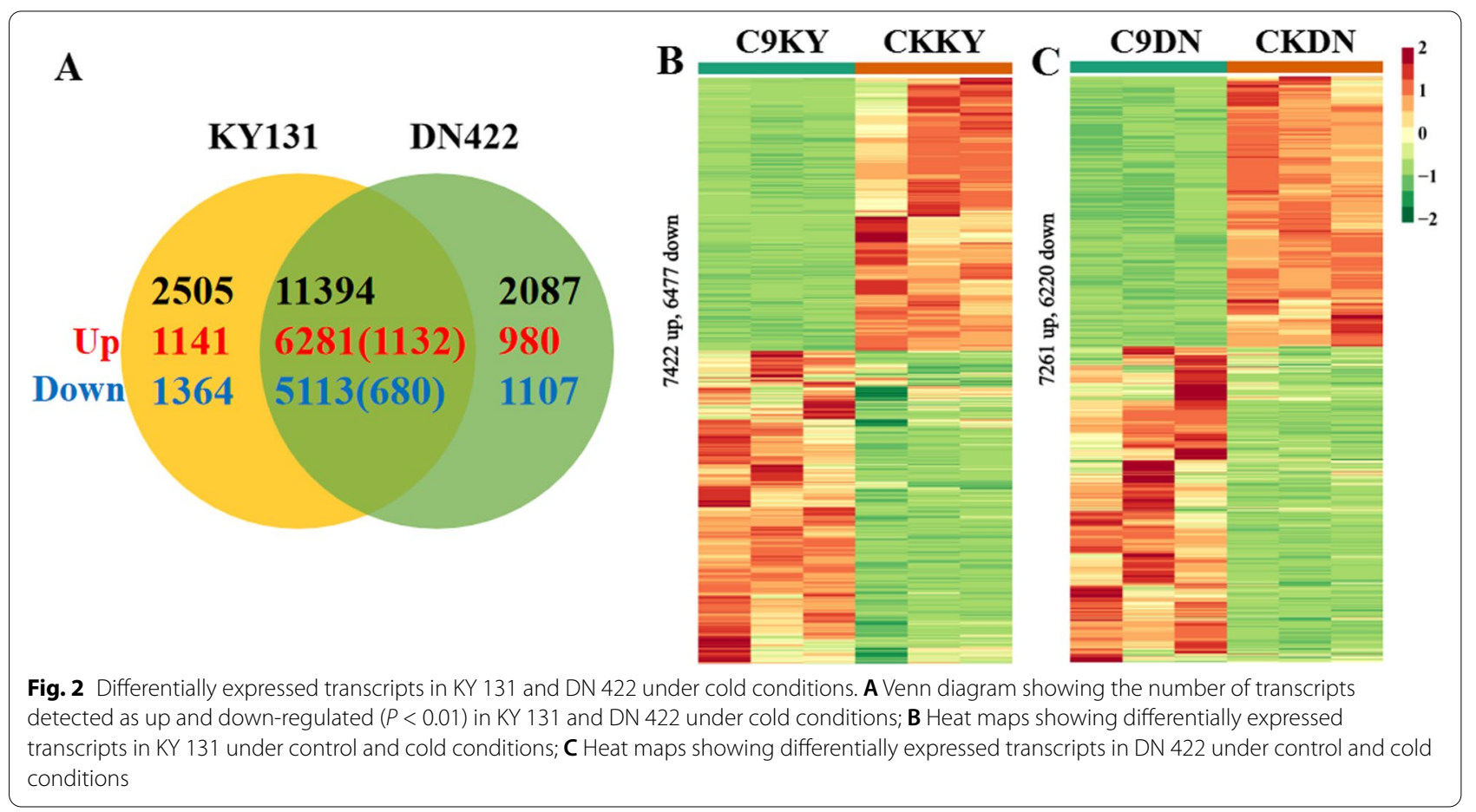




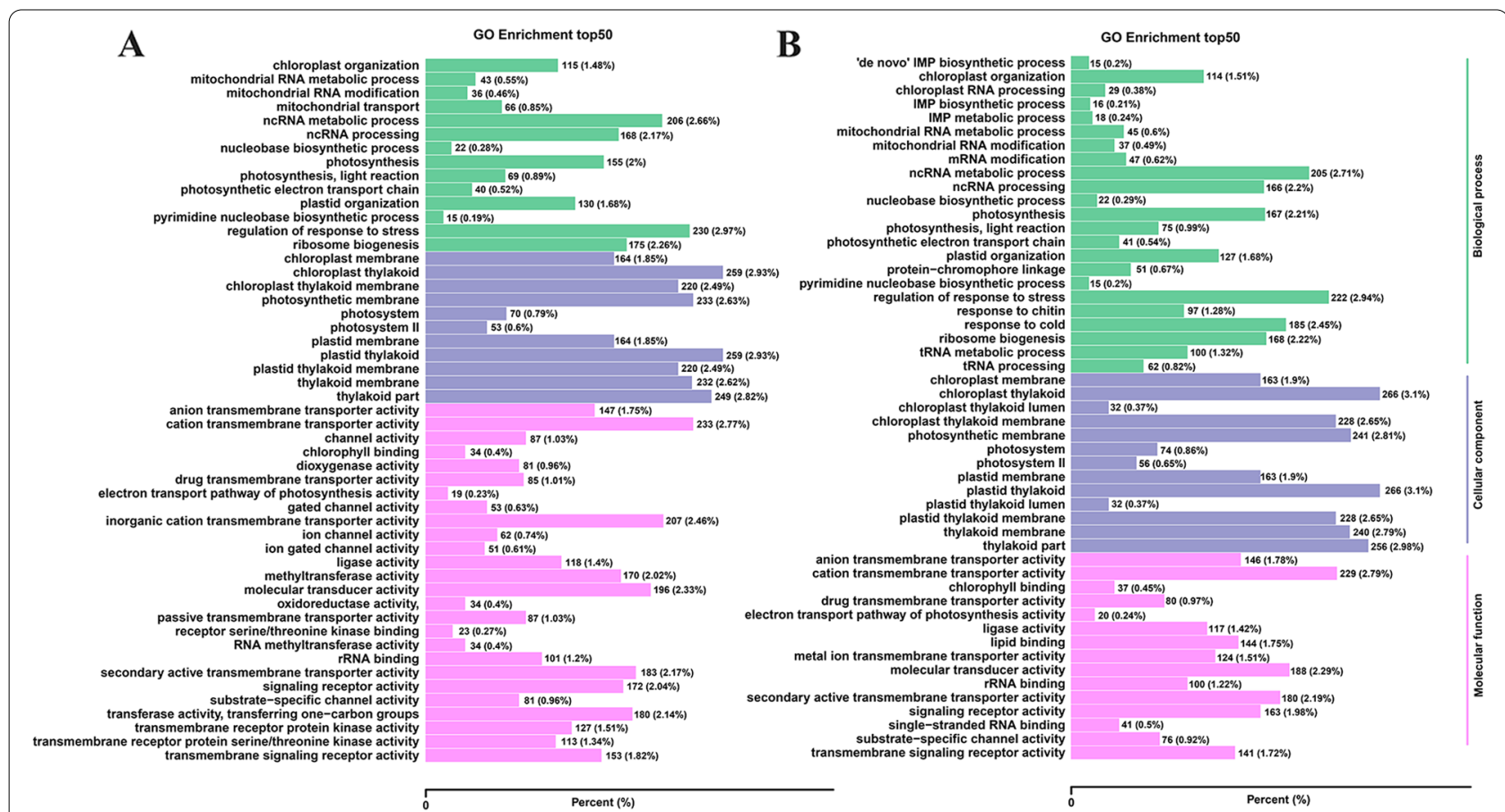

Fig. 3 The Gene Ontology (GO) enrichment analysis under cold conditions. A the top 50 significantly rich GO terms in KY 131 under cold conditions; B the top 50 significantly rich GO terms in DN 422 under cold conditions

were 'photosynthesis', 'ncRNA processing', 'chloroplast organization', 'ncRNA metabolic process', and 'plastid organization', and the specific term enrichment for $\mathrm{KY}$ 131 included 'mitochondrial transport', 'ribosome biogenesis', and 'ribonucleoprotein complex biogenesis. The GO enrichment analysis results showed that the function of the photosynthetic membrane, chloroplast membrane, thylakoid membrane, and other biomembranes, as well as transmembrane substances and signal conduction processes, are significantly affected by low temperature stress, indicating that the stability of biofilms under low temperature stress is closely related to the low-temperature tolerance of rice.

The KEGG pathway enrichment analysis was conducted to determine the biological pathways involved in cold stress (Tables S5, 6). The top 20 pathways were significantly enriched in the CKKY vs. C9KY and CKDN vs. C9DN groups (Fig. 4). These metabolic pathways were mainly enriched in 'carbohydrate metabolism,' 'amino acid metabolism,' 'lipid metabolism', MAPK signaling pathway, plant-pathogen interaction, and stress-resistance-related metabolic pathways (MAPK signaling pathway, plantpathogen interaction). The MAPK signaling pathway plays an important role in plants adapted to cold stress. There were 188 DEGs involved in the MAPK signaling pathway in CKKY vs. C9KY, of which only 184 were also DEGs in CKDN vs. C9DN. In the MAPK signaling pathway in CKKY vs. C9KY, 19 genes were specifically upregulated, and 11 were specifically downregulated (Table S7).

\section{Cold response transcription factors differences in rice cultivars}

Transcription factors (TFs) play important roles in plant growth and response to environmental changes by mediating gene expression. As shown in Fig. 5, under cold stess conditions, 939 TFs were differentially expressed in KY131, and 904 TFs were differentially expressed in DN422. Among them, 135 TFs were specifically expressed in cold-tolerant cultivar KY131, and the expression changes of 137 TFs in KY131 were significantly higher than those in DN422 (FC(KY131/DN422) $\geq 1.5$ ), which genes may play a central role in cold tolerance in rice (Fig. 6). These transcription factors cover a total of 55 transcription factor families, among which AP2/ERF-ERF, bHLH, bZIP, MYB and NACZ transcription factor family members are more, and most of them are up-regulated under cold stress. These results transcriptional regulation plays an important role in rice adaptation to temperature changes and is an important part of rice response to cold stress. 


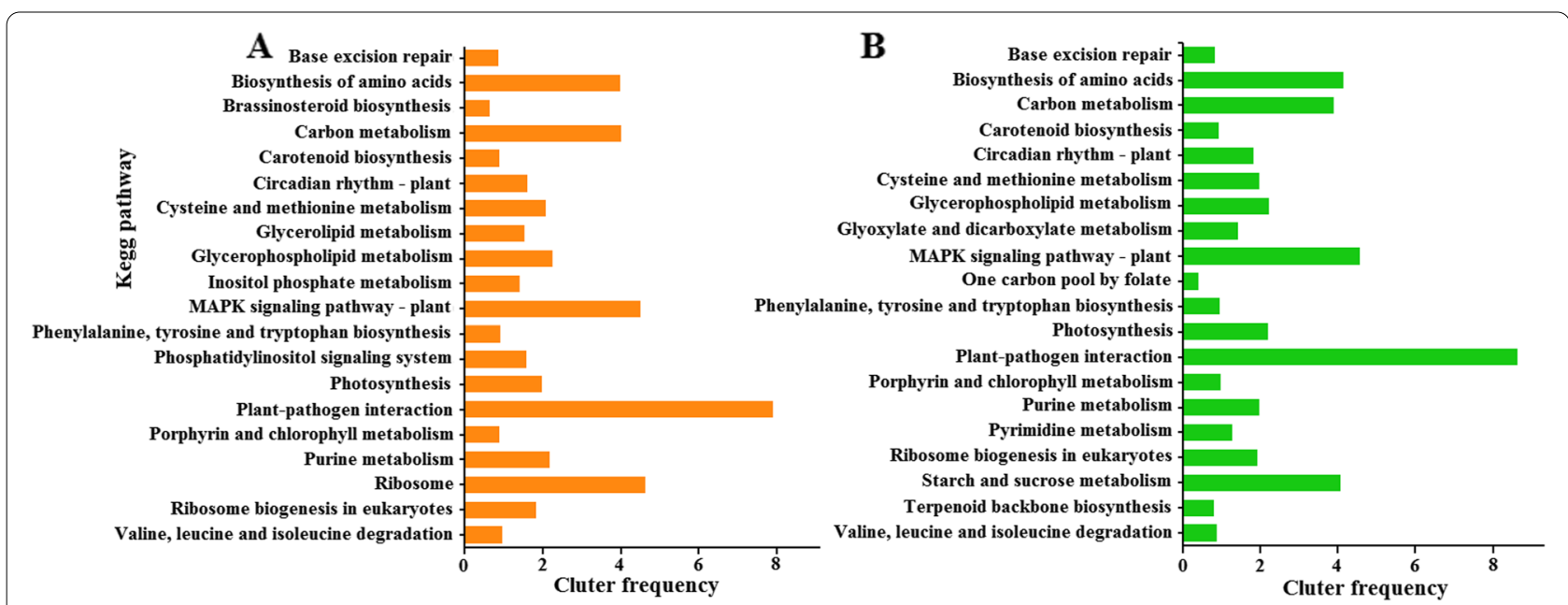

Fig. 4 The Kyoto Encyclopedia of Genes and Genomes (KEGG) enrichment analysis under cold conditions. A the top 20 significantly rich pathways in KY 131 under cold conditions; $\mathbf{B}$ the top 20 significantly rich pathways in DN 422 under cold conditions

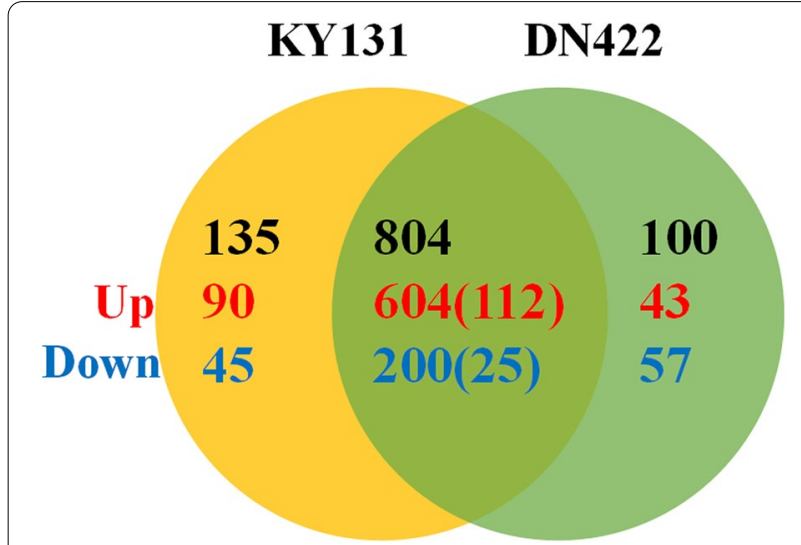

Fig. 5 Transcription factors (TFs) differentially expressed in KY 131 and DN 422 under cold conditions

\section{Lipid metabolism-related genes}

Transcriptional profiling of two rice cultivars indicated that lipid metabolism may play a central role in rice cold tolerance; therefore, we further focused our analyses on the changes in transcription activity of a compiled list of 391 DEGs involved in lipid metabolism. Under cold conditions, 349 DEGs were detected in KY 131, with 238 upregulated and 111 downregulated; 326 DEGs were detected in DN 422, with 228 upregulated and 98 downregulated and of these, 284 DEGs were identified in both varieties, with 199 up-regulated and 85 down-regulated in both varieties, suggesting that lipid metabolism pathways showed significant changes in adaptation to low temperature stress (Fig. 7a). We randomly selected 27 DEGs of lipid metabolism for
qRT-PCR in the two varieties. Correlation analysis showed that the qRT-PCR results were highly correlated with the RNA-seq results, with a Pearson coefficient of 0.96 (Fig. 7b). These results showed that the RNA-Seq data were reliable. The KEGG enrichment analysis showed that DEGs were enriched in 14 metabolic pathways including "Fatty acid biosynthesis," "Fatty acid elongation," and "Fatty acid degradation" in both two varieties (Fig. 7c, d).

\section{Quantitative changes in lipid composition induced by cold stress}

To investigate whether the differential expression of lipid metabolism genes was reflected in differential lipid abundances, the lipidomes of KY 131 and DN 422 were compared quantitatively under cold stress. In our study, a total of 14 lipid headgroup classes and 218 molecular lipid species were detected (Table 1, S8). The total lipid contents of KY 131 and DN 422 were 13061.27 and $14709.55 \mathrm{nmol} \mathrm{mg}^{-1} \mathrm{FW}$ under control conditions and were 15932.01 and $14526.62 \mathrm{nmol} \mathrm{mg}^{-1} \mathrm{FW}$ under cold conditions. Galactolipids, including monogalactosyldiacylglycerol (MGDG), digalactosyldiacylglycerol (DGDG) and sulfoquinovosyldiacylglycerol (SQDG), are the main constituents of chloroplast membranes and photosynthetic complexes, accounting for approximately $66 \%$ of the total lipids. The MGDG content in DN 422 was significantly decreased, while in KY 131 it was significantly increased, which was mainly caused by the significant change in MGDG 34:3 and 36:6. The higher MGDG content can inhibit the reduction of chloroplast membrane lipids and maintain the integrity of the chloroplast 


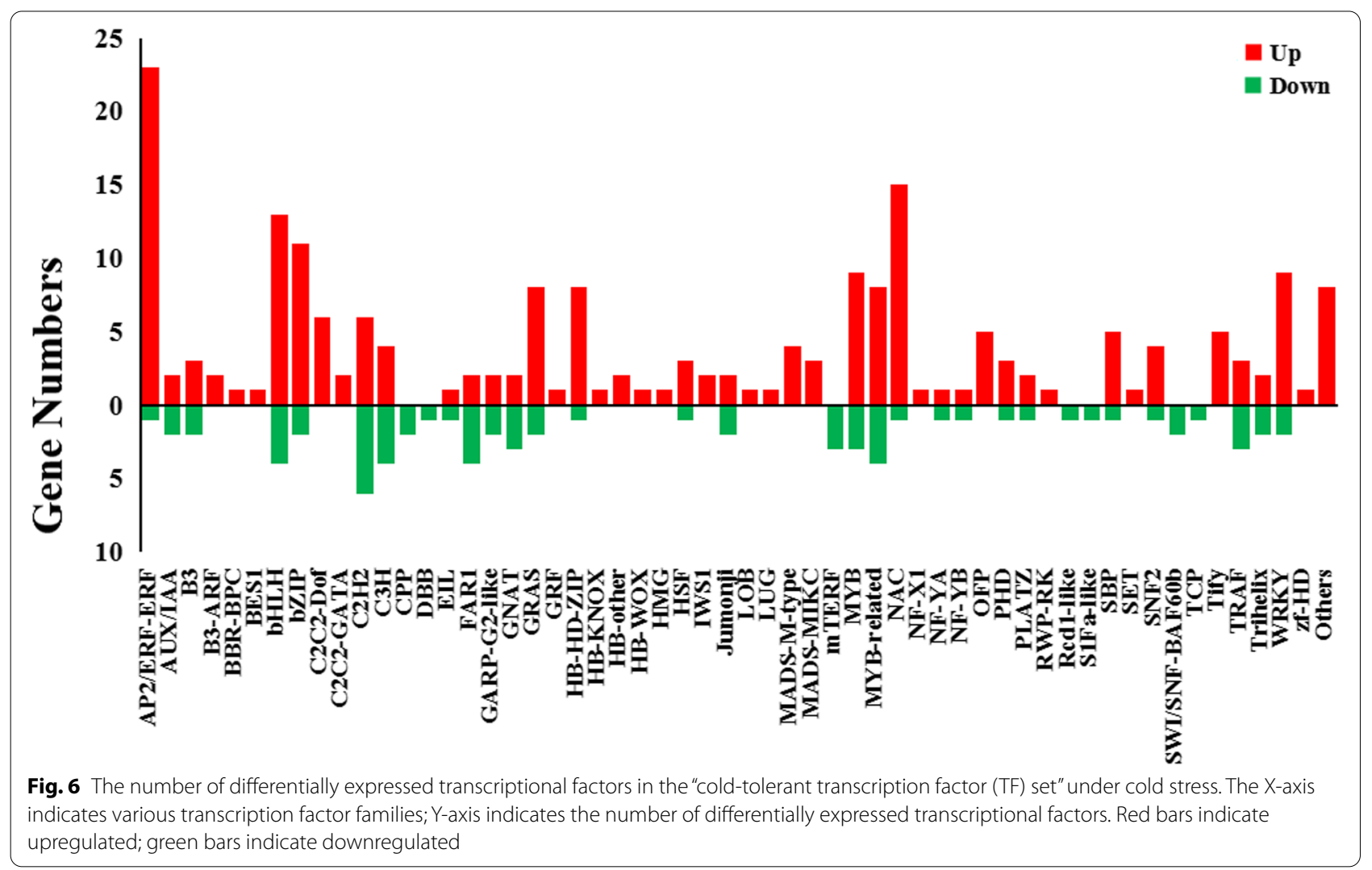

membrane to a certain extent, thereby inhibiting a significant reduction in photosynthesis.

Phospholipids are important structural lipids that make up cell membranes. The content of phospholipids in rice leaves accounts for about $21-24 \%$ of the total lipid content and of these, PG, PC, PE, and phosphatidylinositol (PI) were the most abundant, and PC, PE, and PI were significantly increased in KY 131, with the PC 34:3, 36:5, and C36:6 species contributing the most to the increase in $\mathrm{PC}$, and the PE 34:3 and 36:5 species contributing more to the increase in PE. In this study, two lyso-phospholipids were detected, including lyso-phosphatidylcholine (LPC) and lyso-phosphatidylethanolamine (LPE), which were significantly increased in KY 131 and DN 422 under cold conditions.

In addition to membrane lipids, small amounts of the intermediate lipid DG and the storage lipid triacylglycerol (TG) were also detected. There were 21 DG molecular species in rice leaves and the DG content increased in both varieties under cold conditions. The contents of DG with acyl chains of DG 36:5 and 36:6 were the major contributors to the increased DG. The TG content was significantly reduced in KY 131, and significantly increased in DN 422, under cold conditions, of which TG $52: 4,52: 5,52: 6,54: 5,54: 6,54: 7,54: 8$, and $54: 9$ were the main factors causing this result. An increased DG-TG ratio was observed in KY 131, which indicates that the degradation rate of TG was increased in KY 131 under cold conditions. Cold treatment was able to modulate the accumulation of lipid composition in rice. As such, the cold and control treatment were clearly segregated in distinct quadrants of the biplot based on lipid molecular species composition and accumulation. This segregation was based on principal component analysis (PCA), where $68.9 \%$ of the variability observed in rice lipids could be explained by both $\mathrm{PC} 1$ and $\mathrm{PC} 2$. These results showed that MGDG, PG, PC, PE, LPC, and LPE are the main factors affecting the difference in cold tolerance between $\mathrm{KY}$ 131 and DN 422 (Fig. 8).

\section{Changes in the degree of membrane lipid unsaturation induced by cold stress}

The above analysis results show that under cold stress, most lipid molecules in rice leaves will change to a certain extent, and there are obvious differences among varieties with different cold tolerance. Changes in the content of lipid molecular species can directly affect the fatty acid groups attached to them, changing their saturation, which in turn affects the unsaturation of membrane lipids. Double bond index (DBI) is the average number 


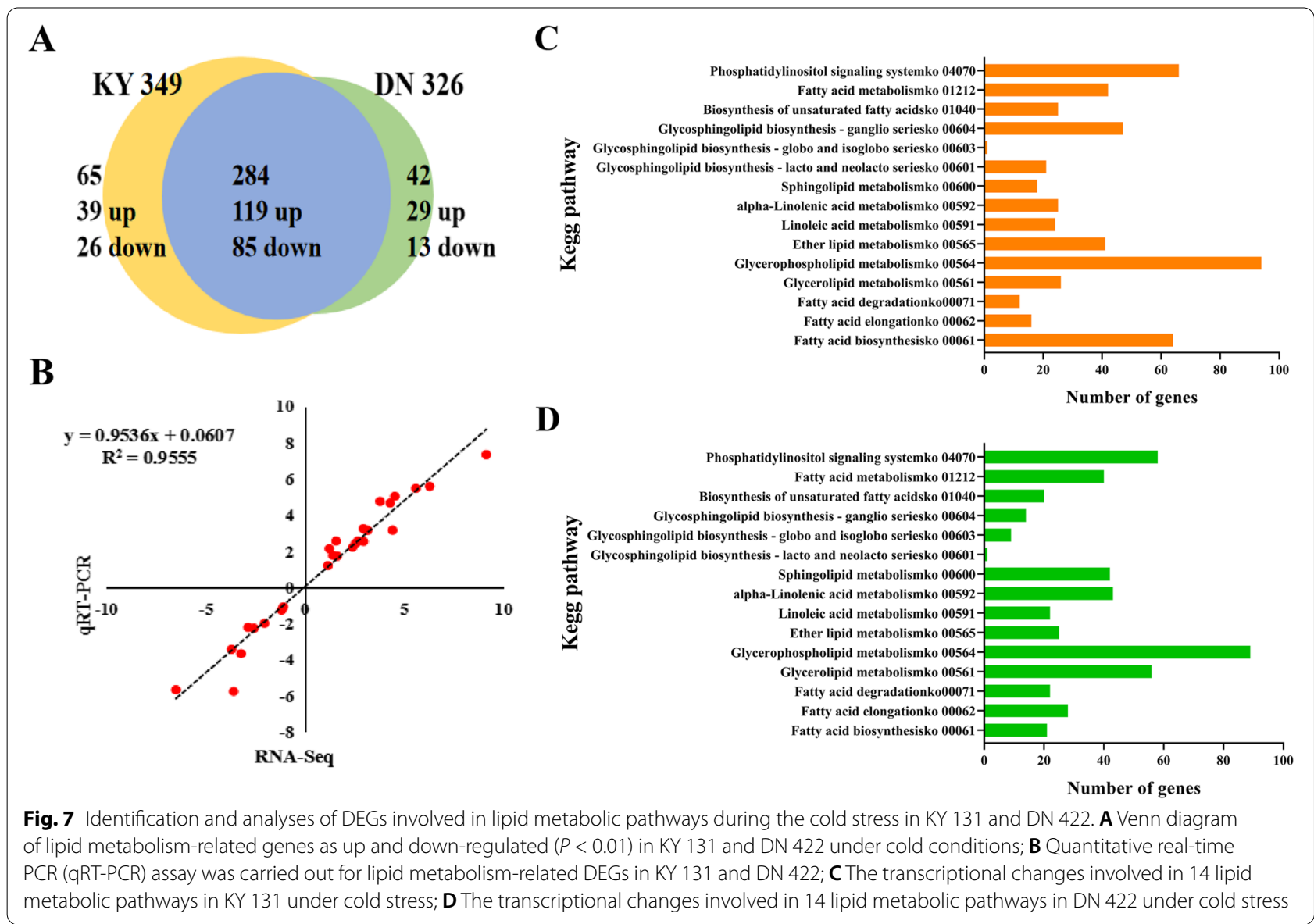

Table 1 The changes of lipid components (nmol g-1 FW) after cold treatments in KY 131 and DN 422. Data are the means \pm SD. Means denoted by the same letter do not differ signifificantly according to Tukey's test $(p<0.05)$

\begin{tabular}{|c|c|c|c|c|c|c|}
\hline \multirow[t]{2}{*}{ Lipid class } & \multicolumn{2}{|l|}{ KY } & \multicolumn{2}{|l|}{ DN } & \multicolumn{2}{|c|}{$\begin{array}{l}\text { Relative change } \\
\text { (RC) }\end{array}$} \\
\hline & Control & Cold & Control & Cold & $\mathrm{KY}$ & DN \\
\hline MGDG & $5084.35 \pm 236.46 b$ & $6796.77 \pm 189.52 \mathrm{a}$ & $6328.62 \pm 534.29 a$ & $5249.45 \pm 259.11 b$ & 33.68 & -17.05 \\
\hline DGDG & $2256.06 \pm 252.23 b$ & $2926.05 \pm 145.14 a$ & $2623.1 \pm 252.67 a$ & $2894.27 \pm 4.65 a$ & 29.7 & 10.34 \\
\hline SQDG & $1121.07 \pm 126.96 a$ & $942.53 \pm 79.75 a$ & $1191.25 \pm 14.00 \mathrm{a}$ & $1080.82 \pm 48.24 a$ & -15.93 & -9.27 \\
\hline PA & $69.44 \pm 8.18 a$ & $52.95 \pm 5.79 a$ & $52.89 \pm 1.11 \mathrm{a}$ & $61.25 \pm 5.99 a$ & -23.75 & 15.81 \\
\hline PC & $309.01 \pm 29.72 b$ & $448.79 \pm 32.48 a$ & $364.89 \pm 35.2 \mathrm{a}$ & $319.34 \pm 17.01 a$ & 45.23 & -12.48 \\
\hline $\mathrm{PE}$ & $469.4 \pm 35.85 b$ & $591.79 \pm 34.2 \mathrm{a}$ & $487.52 \pm 29.78 a$ & $444.07 \pm 24.58 \mathrm{a}$ & 26.07 & -8.91 \\
\hline$P G$ & $1668.29 \pm 89.71 \mathrm{a}$ & $1777.14 \pm 17.5 \mathrm{a}$ & $1740.37 \pm 104.9 a$ & $1685.85 \pm 47.56 b$ & 6.52 & -3.13 \\
\hline $\mathrm{Pl}$ & $626.04 \pm 21.41 b$ & $729.07 \pm 22.99 a$ & $440.92 \pm 41.57 b$ & $528.48 \pm 66.77 a$ & 16.46 & 19.86 \\
\hline $\mathrm{PMeOH}$ & $18.92 \pm 1.06 a$ & $18.36 \pm 0.98 a$ & $21.99 \pm 0.68 a$ & $21 \pm 1.59 a$ & -2.96 & -4.5 \\
\hline LPE & $14.97 \pm 1.09 b$ & $18.64 \pm 1.64 a$ & $26.03 \pm 2.58 \mathrm{~b}$ & $41.83 \pm 3.00 \mathrm{a}$ & 24.52 & 60.7 \\
\hline LPC & $17.24 \pm 1.39 a$ & $16.38 \pm 1.19 a$ & $23.05 \pm 2.42 \mathrm{a}$ & $23.8 \pm 0.36 a$ & -4.99 & 3.25 \\
\hline DG & $856.76 \pm 29.89 b$ & $1264.52 \pm 107.51 \mathrm{a}$ & $1080.69 \pm 92.26 b$ & $1766.78 \pm 26.59 a$ & 47.59 & 63.49 \\
\hline TG & $171.88 \pm 4.30 \mathrm{a}$ & $88.54 \pm 6.00 b$ & $87.91 \pm 7.84 b$ & $133 \pm 2.28 a$ & -48.49 & 51.29 \\
\hline FA & $377.86 \pm 21.73 a$ & $260.47 \pm 9.89$ & $240.32 \pm 19.19 b$ & $276.68 \pm 2.19 a$ & -31.07 & 15.13 \\
\hline Total & $13061.27 \pm 340.00 b$ & $15932.01 \pm 357.56 a$ & $14709.54 \pm 636.42 \mathrm{a}$ & $14526.62 \pm 249.47 a$ & 21.98 & -1.24 \\
\hline
\end{tabular}




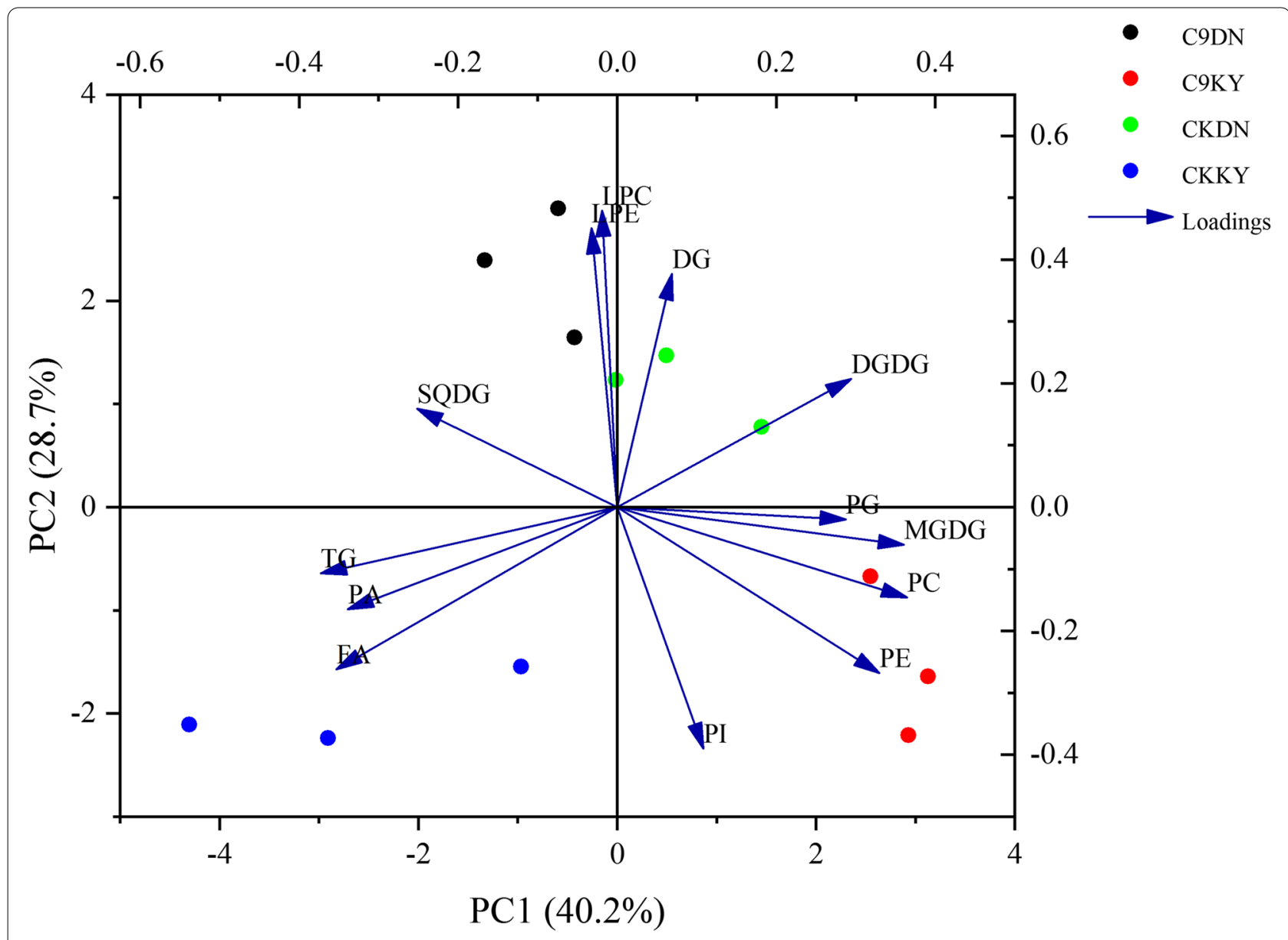

Fig. 8 The principal component analysis (PCA) of lipid components determined on rice cultivars when grown with control and cold conditions

Table 2 Double-bond index (DBI) of membrane lipids after cold treatments in KY 131 and DN 422. DBI = ( $[\mathrm{N} \times \mathrm{mol} \%$ lipid])/100. Data are the means \pm SD. Means denoted by the same letter do not differ signifificantly according to Tukey's test $(p<0.05)$

\begin{tabular}{|c|c|c|c|c|c|c|}
\hline \multirow[t]{2}{*}{ Lipid class } & \multicolumn{2}{|l|}{ KY } & \multicolumn{2}{|l|}{ DN } & \multicolumn{2}{|c|}{ Relative change (RC) } \\
\hline & Control & Cold & Control & Cold & $\mathrm{KY}$ & DN \\
\hline MGDG & $289.28 \pm 13.78 b$ & $386.3 \pm 11.51 a$ & $358.19 \pm 30.8 \mathrm{a}$ & $298.79 \pm 14.82 b$ & 33.54 & -16.58 \\
\hline DGDG & $115.62 \pm 13 b$ & $150.36 \pm 6.82 a$ & $134.22 \pm 12.54 b$ & $147.78 \pm 1.47 a$ & 30.05 & 10.1 \\
\hline SQDG & $49.32 \pm 5.57 a$ & $41.18 \pm 3.19 a$ & $49.1 \pm 1.09 a$ & $45.8 \pm 1.44 b$ & -16.5 & -6.72 \\
\hline PA & $2.26 \pm 0.24 a$ & $1.8 \pm 0.2 b$ & $1.75 \pm 0.07 b$ & $1.97 \pm 0.17 a$ & -20.35 & 12.57 \\
\hline PC & $8.74 \pm 0.87 b$ & $14.04 \pm 0.94 a$ & $10.55 \pm 1.1 \mathrm{a}$ & $9.96 \pm 0.53 a$ & 60.64 & -5.59 \\
\hline PE & $16.45 \pm 1.25 b$ & $21.86 \pm 1.52 \mathrm{a}$ & $17.33 \pm 1.1 \mathrm{a}$ & $15.88 \pm 0.82 \mathrm{a}$ & 32.89 & -8.37 \\
\hline$P G$ & $34.03 \pm 3.28 b$ & $38.18 \pm 0.3 a$ & $34.32 \pm 2.32 \mathrm{a}$ & $35.66 \pm 0.92 a$ & 12.2 & 3.9 \\
\hline $\mathrm{Pl}$ & $5.45 \pm 1 a$ & $5.66 \pm 0.65 a$ & $3.85 \pm 0.41 a$ & $4.2 \pm 0.33 \mathrm{a}$ & 3.85 & 9.09 \\
\hline $\mathrm{PMeOH}$ & $0.55 \pm 0.02 \mathrm{a}$ & $0.55 \pm 0.03 \mathrm{a}$ & $0.65 \pm 0.02 \mathrm{a}$ & $0.64 \pm 0.04 a$ & 0 & -1.54 \\
\hline LPE & $0.24 \pm 0.03 a$ & $0.29 \pm 0.02 \mathrm{a}$ & $0.48 \pm 0.05 b$ & $0.64 \pm 0.05 a$ & 20.83 & 33.33 \\
\hline LPC & $0.27 \pm 0.02 \mathrm{a}$ & $0.23 \pm 0.02 \mathrm{a}$ & $0.37 \pm 0.04 a$ & $0.33 \pm 0.01 a$ & -14.81 & -10.81 \\
\hline DG & $35.67 \pm 0.81 b$ & $58.63 \pm 5.45 a$ & $48.16 \pm 4.44 b$ & $84.13 \pm 2.05 a$ & 64.37 & 74.69 \\
\hline $\mathrm{TG}$ & $10.31 \pm 0.31 a$ & $5.12 \pm 0.39 b$ & $5.33 \pm 0.52 b$ & $8.24 \pm 0.15 a$ & -50.34 & 54.6 \\
\hline FA & $6.98 \pm 0.9 a$ & $4.53 \pm 0.15 b$ & $3.68 \pm 0.27 a$ & $3.72 \pm 0.19 a$ & -35.1 & 1.09 \\
\hline Total & $575.17 \pm 20.72 b$ & $728.72 \pm 18.79 a$ & $667.97 \pm 35.63 a$ & $657.74 \pm 13.14 \mathrm{a}$ & 26.7 & -1.53 \\
\hline
\end{tabular}


of fatty acid double bonds in membrane lipid molecules, and is an important indicator of the total unsaturation of lipids. As shown in Table 2, under cold stress, the DBI of each membrane lipid component in the two rice cultivars changed significantly. The total membrane lipid DBI in KY 131 increased by $26.70 \%$, while the total membrane lipid DBI in DN 422 decreased by $1.53 \%$, indicating that cold-tolerant rice varieties can adapt to cold stress by increasing the membrane lipid unsaturation level. Among the membrane lipid components, MGDG has the highest DBI, which is the main reason for the difference in total membrane lipid unsaturation between KY131 and DN422 under cold stress. In addition, DG, PC, TG, FA, and LPC showed larger RC in DBI ranging from 33.39 to $74.71 \%$, which was another major factor for the increase of total membrane lipid unsaturation.

\section{Changes in free fatty acid components induced by cold stress}

Sixteen FA components were detected in the rice leaves (Table 3). Although the main FA classes were similar, there was great diversity in their contents between the two cultivars under cold stress. The total fatty acid (TFA) content decreased by $31.06 \%$ and increased by $15.13 \%$ in KY 131 and DN 422. Simultaneously, the content of saturated fatty acids (SFAs) was decreased in KY 131, but increased in DN 422. The content of unsaturated fatty acids (UFAs) was decreased in KY 131, but was almost unchanged in DN 422. In general, KY 131 accumu-

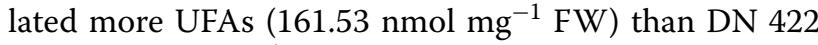
(138.47 $\left.\mathrm{nmol} \mathrm{mg}^{-1} \mathrm{FW}\right)$, and exhibited the genetic characteristic of having more UFAs during the adaptation to low temperature. The main reason for this finding may be that KY 131 had a higher FA 18:3 content under cold conditions. Additionally, under cold conditions, the ratio of UFA to SFA was decreased in both varieties, and the ratio of UFA to SFA in KY 131 was higher than that in DN 422 under both conditions, suggesting that higher levels of unsaturated fatty acids are beneficial for rice to adapt to low temperatures.

\section{Construction of a lipid metabolism regulatory network for cold tolerance in rice}

Combined transcriptome and lipid metabolome analysis results, a schematic diagram is presented to illustrate the lipid-related gene-metabolism network in rice adaptation to cold stress, and to elucidate the molecular regulation mechanism of membrane lipid metabolism in rice cold tolerance. As shown in Fig. 9, under cold stress, the up-regulated expression of CDS1 and PAP2 in rice leaves can hydrolyze PA to produce lipid intermediates DG and CDP-DG, which can prevent plants from excessive

Table 3 The changes of fatty acid components (nmol g-1 FW) after cold treatments in KY 131 and DN 422. Data are the means \pm SD. Means denoted by the same letter do not differ signifificantly according to Tukey's test $(p<0.05)$

\begin{tabular}{|c|c|c|c|c|c|c|}
\hline \multirow[t]{2}{*}{ Fatty acid } & \multicolumn{2}{|l|}{ KY } & \multicolumn{2}{|l|}{ DN } & \multicolumn{2}{|c|}{ Relative change (RC) } \\
\hline & Control & Cold & Control & Cold & $\mathrm{KY}$ & DN \\
\hline FA 14:0 & $1.67 \pm 0.20 a$ & $1.32 \pm 0.07 b$ & $1.19 \pm 0.11 b$ & $1.41 \pm 0.05 \mathrm{a}$ & -20.73 & 18.69 \\
\hline FA 16:0 & $73.28 \pm 6.19 \mathrm{a}$ & $53.90 \pm 4.99 b$ & $62.53 \pm 5.90 b$ & $83.45 \pm 3.99 a$ & -26.45 & 33.46 \\
\hline FA 17:0 & $2.42 \pm 0.25 a$ & $1.94 \pm 0.16 b$ & $2.05 \pm 0.19 b$ & $2.60 \pm 0.11 \mathrm{a}$ & -19.77 & 27.24 \\
\hline FA 18:0 & $31.24 \pm 1.66 \mathrm{a}$ & $24.10 \pm 3.54 b$ & $21.57 \pm 3.24 b$ & $29.22 \pm 4.34 \mathrm{a}$ & -22.85 & 35.45 \\
\hline FA 18:1 & $11.90 \pm 0.89 a$ & $7.09 \pm 0.72 b$ & $10.82 \pm 1.19 a$ & $10.71 \pm 0.49 a$ & -40.42 & -1.03 \\
\hline FA $18: 2$ & $28.20 \pm 5.40 \mathrm{a}$ & $15.65 \pm 0.69 b$ & $19.41 \pm 2.34 \mathrm{a}$ & $20.01 \pm 1.00 \mathrm{a}$ & -44.51 & 3.07 \\
\hline FA $18: 3$ & $209.34 \pm 30.49 a$ & $137.83 \pm 5.04 b$ & $105.54 \pm 6.86 a$ & $106.51 \pm 5.67 \mathrm{a}$ & -34.16 & 0.91 \\
\hline FA 19:1 & $0.27 \pm 0.03 a$ & $0.24 \pm 0.01 \mathrm{a}$ & $0.23 \pm 0.02 b$ & $0.29 \pm 0.01 \mathrm{a}$ & -9.67 & 25.28 \\
\hline FA 20:0 & $4.05 \pm 0.56 a$ & $3.95 \pm 0.15 a$ & $3.34 \pm 0.18 b$ & $4.45 \pm 0.27 a$ & -2.32 & 33.37 \\
\hline FA 20:1 & $0.46 \pm 0.04 a$ & $0.35 \pm 0.03 b$ & $0.39 \pm 0.01 b$ & $0.43 \pm 0.02 \mathrm{a}$ & -24.24 & 9.34 \\
\hline FA 20:2 & $0.37 \pm 0.02 a$ & $0.31 \pm 0.04 a$ & $0.36 \pm 0.02 b$ & $0.45 \pm 0.01 \mathrm{a}$ & -17.07 & 23.77 \\
\hline FA 22:0 & $2.90 \pm 0.18 a$ & $2.78 \pm 0.22 \mathrm{a}$ & $2.84 \pm 0.15 b$ & $3.94 \pm 0.06 a$ & -3.99 & 38.93 \\
\hline FA 22:1 & $0.07 \pm 0.001 \mathrm{a}$ & $0.05 \pm 0.01 b$ & $0.07 \pm 0.01 \mathrm{a}$ & $0.07 \pm 0.03 \mathrm{a}$ & -22.58 & 6.65 \\
\hline FA 22:2 & $0.01 \pm 0.001 \mathrm{a}$ & $0.01 \pm 0.001 \mathrm{a}$ & $0.01 \pm 0.001 \mathrm{a}$ & $0.02 \pm 0.002 \mathrm{a}$ & -23.75 & 9.96 \\
\hline FA 23:0 & $2.05 \pm 0.13 a$ & $2.01 \pm 0.16 a$ & $1.84 \pm 0.07 b$ & $2.51 \pm 0.10 \mathrm{a}$ & -1.98 & 36.77 \\
\hline FA 24:0 & $9.62 \pm 0.74 \mathrm{a}$ & $8.93 \pm 0.97 a b$ & $8.12 \pm 0.15 b$ & $10.60 \pm 0.36 a$ & -7.17 & 30.63 \\
\hline TFA & $377.85 \pm 21.73 a$ & $260.47 \pm 9.87 b$ & $240.30 \pm 19.19 b$ & $276.66 \pm 2.19 a$ & -31.07 & 15.13 \\
\hline UFA & $250.63 \pm 29.54 a$ & $161.53 \pm 5.35 b$ & $136.84 \pm 10.43 a$ & $138.47 \pm 6.22 \mathrm{a}$ & -35.55 & 1.19 \\
\hline SFA & $127.22 \pm 8.71 \mathrm{a}$ & $98.94 \pm 8.56 b$ & $103.46 \pm 8.75 b$ & $138.19 \pm 8.21 \mathrm{a}$ & -22.23 & 33.57 \\
\hline UFA/SFA & $1.97 \pm 0.39 a$ & $1.63 \pm 0.14 a$ & $1.32 \pm 0.01 \mathrm{a}$ & $1.00 \pm 0.10 b$ & -17.26 & -24.24 \\
\hline
\end{tabular}




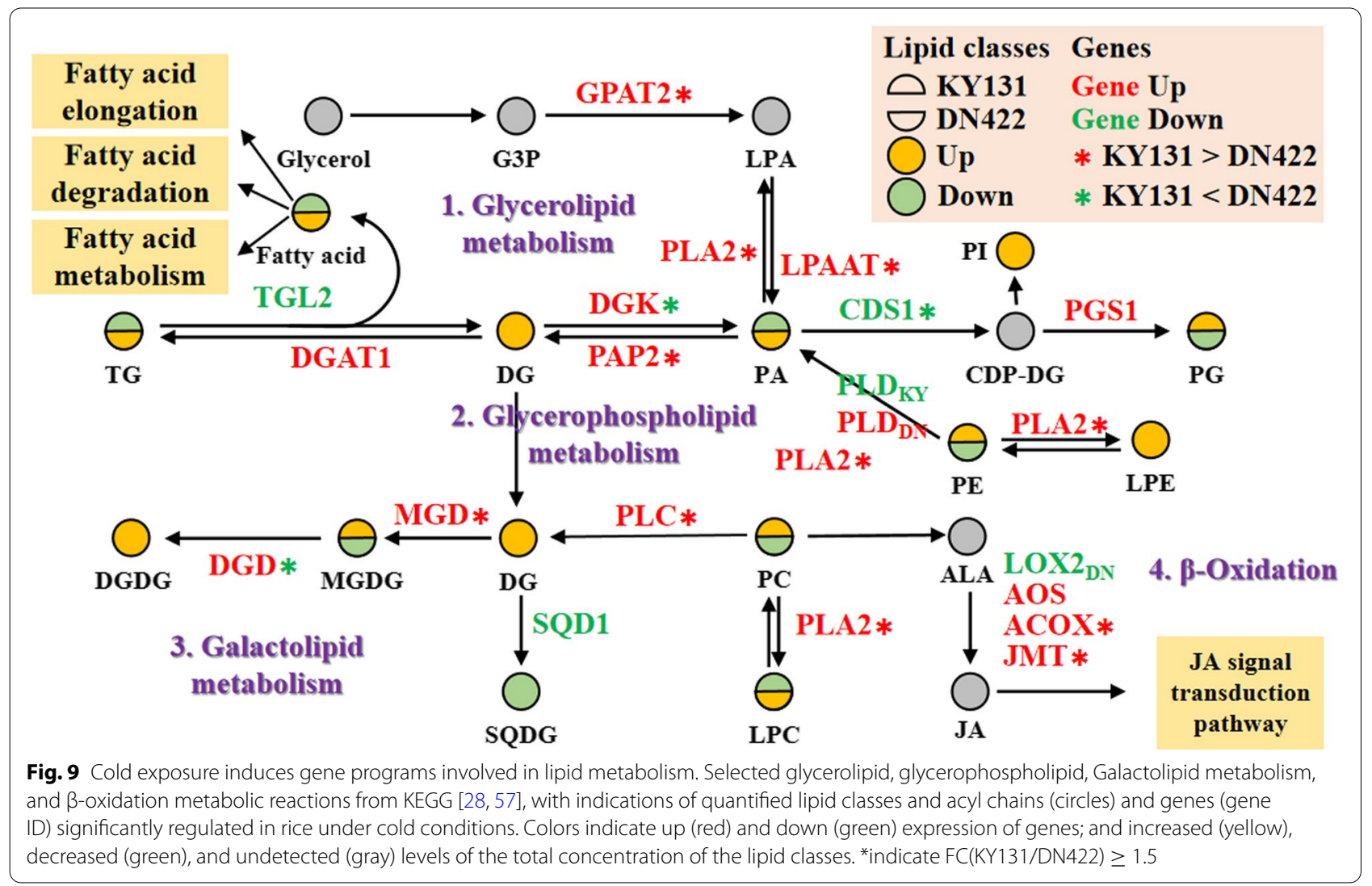

accumulation of PA and cause membrane lipid peroxidation damage. At the same time, the up-regulated expression of of MGD and GLA can directly lead to the increase of MGDG content, significantly increases the unsaturation of membrane lipids, which is crucial to ensure the structural integrity of chloroplast and maintain the normal process of photosynthesis under cold stress. Interestingly, the genes involved in fatty acid $\beta$-oxidation were all significantly up-regulated, which prevent the formation of very long chain fatty acids and promote the production of unsaturated fatty acids such as C16 and C18 by fatty acyl-CoA, thereby increasing the unsaturation of membrane lipids and enhancing cold tolerance. The $\beta$-oxidation process of fatty acids is also a central step in the $\alpha$-linolenic acid (C18:3) metabolic pathway. In addition to increasing the unsaturation of membrane lipids, C18:3 can also be used as a precursor to synthesize JA through the $\alpha$-linolenic acid metabolic pathway. Under cold stress, the expression of key genes (AOS, ACOX, and JMT) on the $\alpha$-linolenic acid metabolic pathway in KY131 was significantly up-regulated, indicating that low temperature stress promoted the synthesis of JA. Therefore, the JA signal transduction pathway may play an important role in cold tolerance in rice.

\section{Discussion}

Cold stress seriously affects the growth, development, yield, and quality of crops, and is a key limiting factor for rice production at higher altitudes and colder agricultural regions [23]. Many molecular studies have been performed on reference rice cultivars, providing important insights into the response to stress, although variability leads to the notion that conclusions are not always translatable to other cultivars with agronomic relevance. Photosynthesis is highly sensitive to cold stress [24] and in this study, we observed that there are large differences between cold-sensitive (DN 422) and cold-tolerant (KY 131) varieties in response to cold stress [25, 26], and there are many differences in the physiology, gene expression, and lipid metabolite changes between the two rice varieties under cold conditions. The photosynthesis-related indicators of the two varieties decreased significantly, and the carbon metabolism pathways such as photosynthesis, starch, and sucrose metabolism, and chlorophyll synthesis and degradation were also significantly enriched under low temperature stress. This study speculated that the decrease in Chl content, $\mathrm{Pn}$, and $\mathrm{Fv} /$ Fm was caused by the damaged photosynthetic membrane, which may reduce carbon assimilation products, 
and in turn, inhibit growth and development [27]. Therefore, it is of critical importance to elucidate the specific mechanisms and promote the genetic improvement of cold tolerance in rice cultivars. The integrated analysis of the rice transcriptome and lipidome allowed us to gain more insight into the lipid metabolism regulation mechanism in response to cold stress, which could be of great practical significance in regulating rice lipid metabolism to improve cold tolerance in rice.

At present, many genes, metabolic pathways, and signaling factors have been identified in some plants during cold stress [28-30]; however, a clear and comprehensive relationship between lipid metabolism and cold tolerance has not been established in rice. Recent studies suggest that carbohydrate metabolism, amino acid metabolism, plant-pathogen interaction, and plant hormone signal transduction pathways are necessary and all play a role in response to low temperature stress [31-33]. In this study, these responses were also significantly enriched in the two rice varieties under cold conditions. The results of the GO enrichment hierarchical analysis showed that the stability of the membrane system was significantly affected in both varieties under cold conditions. Most of the coldstress responsive lipid metabolism genes likely have similar expression patterns in both varieties to cope with cold stress. These results show that the membrane lipid metabolism pathway is indispensable for rice to adapt to cold conditions. Several recent studies have also shown that membrane lipid remodeling can modulate lipid composition, fatty acyl group unsaturation, and membrane fluidity, which have been developed as a key strategy for plants to adapt to low temperature stress $[34,35]$.

This study analyzed the DEGs and differential metabolites in lipid metabolism pathways under cold conditions and showed that most of the reactions in the phospholipid synthesis pathway in the endoplasmic reticulum, the galactolipid synthesis pathway in the chloroplast, and the $\alpha$-linolenic acid metabolic pathway were activated, and most of the genes that catalyzed these reaction steps were upregulated under cold conditions. In plant cells, PA is not only an intermediate product produced by glycerophospholipids, galactolipids, and storage lipid TG, but also an important signal molecule, called "lipid second messenger" which is mainly induced by various biological and abiotic stresses. When encountering abiotic stresses such as low temperature and drought, the content of PA in plants usually increases rapidly, playing the role of signal molecules [13]. However, recent studies have shown that PA, as a polar lipid, lacks a hydrophilic head. Under low temperature stress, excessive accumulation of PA leads to the production of active oxygen free radicals, which trigger membrane lipid peroxidation [36]. In this study, the PA content of the two varieties showed different fates. PA in rice varieties leaves accumulated through three pathways: acylation of OsGPATs and OsLPAAT, hydrolysis of OsPLCs and OsPLDs, and phosphorylation of OsDGKs. When PA accumulates, it is also catalyzed and hydrolyzed by OsPAPs, OsPGS1, and OsCDS1/2 into DG and CDP-DAG, and glycerophospholipids are produced under the action of various phospholipid synthases such as PISD, PCYT1/2, and EPT1. The downregulation of OsCDS1 (LOC_OsO2g03460) and OsPAP2 (LOC_Os08g27030) caused excessive accumulation of PA, and subsequently reduced the content of PC, $\mathrm{PE}$, and PG in DN 422. PG is the only phospholipid present in the chloroplast membrane and almost all PGs in plants are distributed in the chloroplast. Although PG occupies a relatively low proportion of membrane lipids, it is the main factor that determines the phase change of membrane lipids and increases fatty acid saturation. Related studies have shown that PG can quickly accumulate unsaturated fatty acids or unsaturated phospholipid molecules when plants adapt to low temperature stress, which is one of the factors necessary for plants to maintain a certain cold tolerance $[37,38]$. Therefore, the upregulated expression of OsCDS1 and OsPAP2 under low temperature stress can prevent peroxidation damage caused by excessive accumulation of PA, while rapidly increasing PG to maintain membrane lipid stability.

Galactolipids are the most abundant lipids in rice leaves, accounting for approximately $66 \%$ of the total lipid content. It mainly includes three components: MGDG, DGDG, and SQDG. In plants, almost all galactolipids are distributed in the thylakoids. Generally, when plants are exposed to low temperature, drought, and salt stress, the MGDG content in their bodies is significantly reduced [37, 39, 40]. Moellering et al. [41] showed that the decrease in MGDG content under low temperature stress is due to its degradation; that is, the abundant galactose residues in MGDG are transferred to other lipid receptors under the action of the glycosyl hydrolase SFR2 in the final production of oligogalactolipids and DAG. In cold-tolerant varieties, the significant increase in MGDG content may be due to the upregulated expression of OsGLA (LOC_Os03g56070), especially the significant increase in MGDG 34:3 and 36:6 content, which not only increased the content of thylakoid membrane lipids but also was increased by reducing the unsaturation of the thylakoid membrane lipid, resulting in less exposure of the thylakoid membrane to cold stress, thereby maintaining a certain degree of photosynthesis. Concurrently, this study also found that another thylakoid membrane lipid component DGDG increased significantly, especially the content of polyunsaturated molecular species such as DGDG C36:6, C36:5-DGDG, and C34:3, which increased the most. To a certain extent, the fluidity and 
integrity of thylakoid membrane lipids are maintained, thereby ensuring normal photosynthesis. Further studies have found that the increase in DGDG content is mainly caused by the significant upregulation of OsMGD and $O s D G D 1$. In Arabidopsis, the knockout of $D G D 1$ hinders the formation of membrane light-harvesting complex II (LHCII) and reduces the stability of PSI. The chlorophyll fluorescence lifetime was significantly shortened [42]. Therefore, the increase in DGDG content caused by the up-regulation of OsMGD and OsDGD1 in rice leaves under low temperature stress is essential to ensure the structural integrity of chloroplasts and maintain the normal progress of photosynthesis.

In addition to membrane lipid metabolism, fatty acid metabolism also plays an important regulatory role in the adaptation of rice to low-temperature stress. Previous studies have shown that since short-chain fatty acids and unsaturated fatty acids have lower melting points, plants can appropriately shorten the length of the fatty acid carbon chain and increase the number of fatty acid double bonds to inhibit the phase transition of membrane lipids under low temperature stress, thereby maintaining cell membrane fluidity [43]. This study demonstrated that under low-temperature conditions, cold-tolerant varieties have higher membrane lipid unsaturation and unsaturated fatty acid content. This enables the plant to maintain a certain degree of stability and fluidity of the membrane system under cold stress. Fatty acid $\beta$-oxidation is the main form of fatty acid degradation in plants and is catalyzed by a multienzyme complex, including ACOX, MFP, and ACAA, to decrease the length of the carbon chain of fatty acids [44]. In the present study, OsACOX1 (LOC_Os03g53630) and OsACOX4 (LOC_Os05g07090) were significantly upregulated in both varieties and activated the $\beta$-oxidation pathway of fatty acids under cold stress. Fatty acid $\beta$-oxidation is the sole pathway for the metabolic breakdown of fatty acids that generate energy and carbon skeletons in plants and plays an important role in plant growth, development, and cellular homeostasis $[45,46]$. Fatty acid $\beta$-oxidation is also the central component of $\alpha$-linolenic acid (C18:3) metabolism. In the $\alpha$-linolenic acid metabolic pathway, C18:3 produces 12-oxo-phytodienoic acid (OPDA) under the action of lipoxygenase (LOX), hydroperoxide dehydratase (AOS), and cetyl-CoA acyltransferase (fadA), and then produce JA through three fatty acid $\beta$-oxidation cycles. In Arabidopsis thaliana, JA can positively regulate the $\mathrm{C}$-repeat-binding factors $(\mathrm{CBF})$ pathway to increase the accumulation of cryoprotective compounds under low temperature stress and interacts with other plant hormones to regulate stomatal closure and maintain the normal progress of light action [47, 48]. These results indicate that rice may improve its tolerance through active JA metabolism and signal transduction when exposed to cold stress.

In this study, these results provide a holistic view of lipid metabolism-related genes and metabolism in response to cold stress. PA, MGDG, DGDG, and FA play important roles in maintaining membrane stability and mobility under cold conditions. Changes in the expression of lipid metabolism-related genes (OsCDS1, OsPAP2, OsGLA OsMGD, and OsDGD1) alter the rice PA, MGDG, DGDG, and FA content to adapt to cold stress.

\section{Materials and methods}

Plant material, cold treatment, and sample preparation

The experiment was conducted at the Rice Research Institute of Northeast Agricultural University in 2020. Kongyu 131 (KY 131), a cold-tolerant rice cultivar, and Dongnong 422 (DN 422), a cold-sensitive rice cultivar, were selected. Rice seeds of the same size, plump, and vigorous were selected and sterilized with $1 \%$ sodium hypochlorite solution for $30 \mathrm{~min}$, soaked in clean water at room temperature for $24 \mathrm{~h}$, and then the paper bed sprouting method was used to cultivate them in the dark at $32{ }^{\circ} \mathrm{C}$ for $48 \mathrm{~h}$. Well-germinated seeds were selected and sown in high-temperature sterilized sand and cultivated in an artificial climate room. During this period, $1 / 2$ Hoagland nutrient solution was rationed daily. The culture conditions in the control temperature climate chamber were $25^{\circ} \mathrm{C}, 10 \mathrm{~h}$ day $/ 14 \mathrm{~h}$ night. At the threeleaf heart stage, the seedlings were transferred to the control temperature and low temperature climate room, and the culture conditions in the low-temperature climate chamber were $10{ }^{\circ} \mathrm{C}, 10 \mathrm{~h}$ day/14 h night. The cold treatment method was slightly modified according to a previous study [26]. Sampling was carried out after nine days of control (CK) and cold (C9) treatments. CKKY and CKDN represent KY131 and DN422 under control conditions; C9KY and C9DN represent KY131 and DN422 under cold treatment conditions. Three rice plants with the same growth were selected, and the rice leaves were cut with sterilized scissors, wrapped in foil paper, marked and placed in liquid nitrogen immediately, and then placed at $-80^{\circ} \mathrm{C}$ in a refrigerator for later use.

\section{Measurement of leaf relative water content, electrolyte leakage, malondialdehyde content, and photosynthetic characteristics}

The leaves were harvested after nine days of treatment. The relative water content of the leaves was determined as described by Wang et al. [49]. Leaf electrolyte leakage was determined using an electrical conductivity meter (DDSJ-308 F, Shanghai, China) according to the method described by Song et al. [50]. Malondialdehyde 
content was measured as described by Chen et al. [54]. Nine days after the low-temperature treatment started, the net photosynthetic rate $(\mathrm{Pn})$ was measured on the penultimate leaves using a CIRAS-3 portable photosynthetic instrument (PP Systems, USA) [52]. The fluorescence parameters $\mathrm{Fv} / \mathrm{Fm}$ and chlorophyll (Chl) fluorescence images of the penultimate leaves were analyzed after 30 min of dark adaptation using fluorescence measurements with a HITACHI F-7000 fluorescence spectrophotometer (Tokyo, Japan) [53]. Chlorophyll (Chl) content was analyzed as previously described by Li et al. [54].

\section{RNA extraction and transcriptome sequencing analysis}

Three samples of rice from the penultimate leaf under the control and low temperature treatment were measured for nine days; three biological replicates per sample were used for the transcriptome analyses. Total RNA was extracted using TRIzol reagent (Invitrogen, Carlsbad, CA, USA). After total RNA was extracted, mRNA was purified using NucleoSpin RNA Clean-up (MACHEREYNAGEL, Düren, Germany) according to the manufacturer's instructions. The purified mRNA was fragmented into short fragments using fragmentation buffer and reverse-transcribed into cDNA using random hexamer primers. Second-strand cDNA was synthesized using DNA polymerase I, RNase $\mathrm{H}$, dNTPs, and buffer. The cDNA fragments were purified using a QiaQuick PCR extraction kit, end repaired, poly(A) was added, and the fragments were ligated to Illumina sequencing adapters. The ligation products were size-selected by $1 \%$ agarose gel electrophoresis, PCR-amplified, and sequenced using an Illumina HiSeqTM 2500 (Wuhan, China). The transcripts were assembled using Cufflinks to obtain the known and new transcripts. Before data analysis, we conducted strict quality control on the data to ensure that these reads were of high enough quality to ensure the accuracy of subsequent analysis. The filtering criteria were as follows: (1) Remove reads with adapters; (2) When the $\mathrm{N}$ content in any sequencing read exceeds $10 \%$ of the base number of the read, remove the paired reads; (3) When the number of low-quality $(\mathrm{Q} \leq 20)$ bases contained in any sequencing read exceeded $50 \%$ of the bases of the read, the paired reads were removed. Then, differential expression analysis across samples was conducted to obtain DEGs (false discovery rate $[F D R]<0.05$, $|\log 2 \mathrm{FC}|>1)$. Genes were annotated against the Gene Ontology (GO) (Available online: http://www.r-project. org/) and Kyoto Encyclopedia of Genes and Genomes (KEGG) (Available online: http://kobas.cbi.pku.edu.cn/) databases to obtain their functions. Significant functional categories and metabolic pathways were identified within differentially expressed genes, with FDR $\leq 0.05$.

\section{Confirmation of lipid metabolism-related genes in the transcriptome using qRT-PCR analysis}

The purified total RNA was reverse-transcribed using Prime Script RT Master Mix (Takara Bio Inc., Shiga, Japan). Real-time PCR was performed using SYBR Premix Ex Taq II (Takara Bio Inc., Shiga, Japan) in the Applied Biosystems QuantStudio 3 (Thermo Fisher Scientific, Germany). OsACTIN1 was used as a reference control. Relative quantification analysis was performed using the comparative CT method. Correlation coefficients for RNA-Seq and qRT-PCR data were plotted and calculated using the Origin 2018 (OriginLab, Northampton, MA, USA) mapping software. The primers used are listed in Table S9.

\section{Lipid extraction and liquid chromatography electrospray ionization tandem mass spectrometry (LC-ESI-MS/MS) analysis}

The cut rice leaf samples of approximately $250 \mathrm{mg}$ were added with $80 \mu \mathrm{L}$ of whole fat internal standard (both at a concentration of $10 \mu \mathrm{g} / \mathrm{mL}$ ), and $2 \mathrm{~mL}$ of methanol. Then $2 \mathrm{~mL}$ of dichloromethane was added, and vortexed for $1 \mathrm{~h}$. This was followed by the addition of $2 \mathrm{~mL}$ of dichloromethane, $1.6 \mathrm{~mL}$ of ultrapure water, vortexing, and centrifugation, removing the supernatant, adding $4 \mathrm{ml}$ of dichloromethane to extract the remaining supernatant, repeating twice, combining the supernatants, blowing with nitrogen, and reconstituting with $1 \mathrm{~mL}$ of isopropanol. Then passed through the $0.22 \mu \mathrm{m}$ organic filter membrane and get on the machine. Lipidomic analysis was performed on an $A B$ Sciex Triple 4000 quadruple electrospray ionization mass spectrometer (ESI/MS) coupled with a Shimadzu UPLC LC-20 A (Shimadzu, Kyoto, Japan). The specific methods were previously described by Gwak et al. [55]. Based on the high-resolution QTOF mass spectrum, the precise molecular weight of the characteristic peaks in the sample was obtained and compared with the database information to obtain the qualitative results of the metabolites. The quantitative results of metabolites were obtained based on the ion signal intensity (CPS) of the characteristic peak in the mass spectrum. MSDIAL ver. 4.00, PeakView 2.1, MultiQuant 3.0.2, and other software were used to complete the qualitative and quantitative lipids.

\section{Statistical analysis}

For experimental variables, one-way of variance (ANOVA) was applied to assess differences among treatments with SPSS 22.0 (Softonic International, Barcelona, Spain) software. Significant differences $(p<0.05)$ 
between treatments are indicated by different letters according to Fisher's LSD. Principal component analysis (PCA) [56] and graphs were employed with Origin 2018 software (OriginLab, Northampton, MA, USA).

\section{Supplementary Information}

The online version contains supplementary material available at https:/doi. org/10.1186/s12870-022-03468-1.

Additional file 1. Differentially expressed genes (DEGs) in KY 131 under cold stress.

Additional file 2. Differentially expressed genes (DEGs) in DN 422 under cold stress.

Additional file 3. Go enrichment analysis of differentially expressed genes (DEGs) in KY 131 under cold stress.

Additional file 4. Go enrichment analysis of differentially expressed genes (DEGs) in DN 422 under cold stress.

Additional file 5. KEGG enrichment analysis of differentially expressed genes (DEGs) in KY 131 under cold stress.

Additional file 6. KEGG enrichment analysis of differentially expressed genes (DEGs) in DN 422 under cold stress.

Additional file 7. The response of the MAPK signaling pathway of two varieties to cold stress.

Additional file 8. Quantitative changes in lipid composition induced by cold stress.

Additional file 9. Sequences of primers used in this study.

\section{Acknowledgements}

Thanks Wuhan Metwell Biotechnology Co., Ltd. for the technical support.

\section{Statement}

The rice seeds used in this study were collected and preserved by the Rice Research Institute of Northeast Agricultural University, and the collection and use of these seeds completely followed the IUCN Policy Statement on Research Involving Species at Risk of Extinction and the Convention on the Trade in Endangered Species of Wild Fauna and Flora.

\section{Authors' contributions}

H.L. and W.X. designed the study and provided experimental materials. W.X analyzed the results and prepared the figures and tables. W.X. wrote the paper. All authors discussed the results and commented on the manuscript. All authors have read and approved the final manuscript.

\section{Funding}

This work was supported by grants from the China Postdoctoral Science Foundation (Grant No. 2021M693792) and the "Breeding of high quality and resistant rice varieties" (Grant No. 2020ZX16B01) a major scientifific and technological project of "Hundreds and Thousands" in Heilongjiang Province, China, and the China.

\section{Availability of data and materials}

The datasets generated for this study can be found in the SRA accession number: SRR15076606, SRR15076607, SRR15076608, SRR15076609, SRR150766011, SRR150766012, SRR15076619, SRR15076620, SRR15076599, SRR15076600, SRR15076601, SRR15076602

\section{Declarations}

Ethics approval and consent to participate

Not applicable.

\section{Consent for publication}

Not applicable.

\section{Competing interests}

The authors declare no conflicts of interest.

Received: 8 July 2021 Accepted: 9 February 2022

Published online: 02 March 2022

\section{References}

1. Li H, Hu B, Chu C. Nitrogen use efficiency in crops: lessons from Arabidopsis and rice. Journal of Experimental Botany. 2017; 68: 2477-2488.

2. Liu C, Schlappi M, Mao B, Wang W, Wang A, Chu C. The bZIP73 transcription factor controls rice cold tolerance at the reproductive stage. Plant Biotechnol. J. 2019; 17: 1834-1849.

3. Jena K, Kim S, Suh J, Yang C, Kim Y. Identification of coldtolerant breeding lines by quantitative trait loci associated with cold tolerance in rice. Crop Sci. 2012; 52: 517-523.

4. Almadanim M, AlexandreBRosa M, Sapeta H, LeitaoARamalho J. Rice calcium-dependent protein kinase OsCPK17 targets plasma membrane intrinsic protein and sucrose-phosphate synthase and is required for a proper cold stress response. Plant Cell Environ. 2017;40:1197-213.

5. Kim K, Cheong Y, Grant J, Pandey G, Luan S. CIPK3 a calcium sensorassociated protein kinase that regulates abscisic acid and cold signal transduction in Arabidopsis. Plant Cell. 2003:15:411-23.

6. Yuan P, Yang T, Poovaiah B. Calcium signaling-mediated plant response to cold stress. Int. J. Mol. Sci. 2018; 19: e3896.

7. Cai G, Wang G, Wang L, Pan J, Liu Y, Li D. Zm MKK1 a novel group a mitogen -activated protein kinase kinase gene maize conferred chilling stress tolerance and was involved in pathogen defense in transgenic tobacco. Plant Science. 2014; 214: 57-73.

8. Cui L, Feng K, Wang M, Wang M, Deng P, Song W, Nie X. Genome-wide identification phylogeny and expression analysis of AP2/ERF transcription factors family in Brachypodium distachyon. BMC Genomics. 2016; 17636.

9. Baldoni E, Genga A, Medici A, Coraggio I, Locatelli F. The Os MYB4 gene family: stress response and transcriptional auto-regulation mechanisms. Biol Plant. 2013;57:691-700.

10. Licausi F, Masaru OT, Perata P. APETALA2/Ethylene Responsive Factor (AP2/ERF) transcription factors: Mediators of stress responses and developmental programs. New Phytologist. 2013;199:639-49.

11. Sun X,L, Li Y, Cai H, Bai X, Ji W, Ji Z, Zhu Y. Arabidopsis bZIP1 transcription factor binding to ABRE cis-element regulates abscisic acid signaltransduction. Acta Agronomica Sinica. 2011; 37: 612-619.

12. Li Q, Zheng Q, Shen W, Cram D, Fowler D, Wei Y. Understanding the biochemical basis of temperature-induced lipid pathway adjustments in plants. Plant Cell. 2015; 27: 86-103.

13. Cantrel C, Vazquez T, Puyaubert J, Reze N, Lesch M, Kaiser W, Dutilleul C, Guillas I, Zachowski A, Baudouin E. Nitric oxide participates in coldresponsive phosphosphingolipid formation and gene expression in Arabidopsis thaliana. New Phytologist. 2011;189:415-27.

14. Peppino MM, Reyna M, Meringer MV, Racagni GE, Villasuso AL. Lipid signalling mediated by PLD/PA modulates proline and $\mathrm{H} 2 \mathrm{O} 2$ levels in barley seedlings exposed to short- and long-term chilling stress. Plant Physiol Biochem 2017:113:149-60.

15. Arisz SA, Wijk RV, Roels W, Zhu JK, Haring MA, MunnikT. Rapid phosphatidic acid accumulation in response to low temperaturestress in Arabidopsis is generated through diacylglycerol kinase. Front. PlantSci. 2013; 4:

16. Tan W, Yang Y, Zhou Y, Huang L, Xu L, Chen Q, Yu L, Xiao S. DIACYLGLYCEROL ACYLTRANSFERASE and DIACYLGLYCEROL KINASE modulate triacylglycerol and phosphatidic acid production in the plant response to freezing stress. Plant Physiol. 2018;177:1303-18.

17. Dubots E, Botte C, Boudiere L, Yamaryo BY, Jouhet J, Marechal E. Role of phosphatidic acid in plant galactolipid synthesis. Biochimie, 2011; 94: 86-93.

18. Gwak Y, Hwang Y, Wang B, Kim M, Jeong J, Lee C, Hu Q, Han D, Jin E. Comparative analyses of lipidomes and transcriptomes reveal a concerted action of multiple defensive systems against photooxidative stress in Haematococcus pluvialis. J Exp Bot. 2014:65:4317-34.

19. Barrero-Sicilia C, Silvestre S, Haslam RP, Michaelson LV. Lipid remodelling: Unravelling the response to cold stress in Arabidopsis and its extremophile relative Eutrema salsugineum. Plant Sci. 2017; 263: 194-200. 
20. Noblet A, Leymarie J, Bailly C. Chilling temperature remodels phospholipidome of Zea mays seeds during imbibition. Sci Rep. 2017;7:8886.

21. Suzuki S, Awai K, Ishihara A, Yamauchi K. Cold temperature blocks thyroid hormone-induced changes in lipid and energy metabolism in the liver of Lithobates catesbeianus tadpoles. Cell Biosci. 2016; 6: 19.

22. Almadanim M, Alexandre B, Rosa M, Sapeta H, Leitao A, Ramalho J, Lam T, Negrao S, Abreu I, Oliveira M. Rice calcium-dependent protein kinase Os CPK17 targets plasma membrane intrinsic protein and sucrose-phosphate synthase and is required for a proper cold stress response. Plant Cell Environ. 2017:40:1197-213.

23. Yang L, Lei L, Li P, Wang J, Zou D. Identification of candidate genes conferring cold tolerance to rice (oryza sativa I.) at the bud-bursting stage using bulk segregant analysis sequencing and linkage mapping. Front Plant Sci. 2021;12:647239.

24. Peng $X$, Teng L, Yan $X$, Zhao M, Shen $S$. The cold responsive mechanism of the paper mulberry: Decreased photosynthesis capacity and increased starch accumulation. BMC Genomics. 2015; 16: 898.

25. Yang L, Zhao H, Wang J, Liu H, Sun J, Guo L. Genomic dissection of rice yield traits under low temperature across multi-environments. Euphytica. 2017; 213.

26. Yang L, Liu H, Lei L, Zhao H, Wang J, Li N. Identification of qtls controlling low-temperature germinability and cold tolerance at the seedling stage in rice (oryza sativa l.). Euphytica. 2018; 214: 13.

27. Weiszmann J, Furtauer L, Weckwerth W, Nagele T. Vacuolar sucrose cleavage prevents limitation of cytosolic carbohydrate metabolism and stabilizes photosynthesis under abiotic stress. FEBS J. 2018; 285: 4082-4098.

28. Zhong C, Jiang C, Ren J, Dong J, Yu H. An advanced lipid metabolism system revealed by transcriptomic and lipidomic analyses plays a central role in peanut cold tolerance. Frontiers in Plant Science. 2020; 11: 1110.

29. Shen C, Li D, He R, Fang Z, Xia Y, Gao J. Comparative transcriptome analysis of rna-seq data for cold-tolerant and cold-sensitive rice genotypes under cold stress. J Plant Biol. 2014;57:337-48.

30. Dong J, Zhao J, Zhang S, Yang T, Liu Q, Mao X. Physiological and genomewide gene expression analyses of cold-induced leaf rolling at the seedling stage in rice(oryza sativa l.). Crop J. 2019;7:431-43.

31. Xin H, Xianchao N, Pan X, Wei L, Min Y, Yu K, Lunwen Q, Wei H. Comparative transcriptome analyses revealed conserved and novel responses to cold and freezing stress in Brassica napus L. G3. 2019; 9: 2723-737.

32. Ma L, Coulter J, A, Liu L, Zhao Y, Chang Y, Pu Y. Transcriptome analysis reveals key cold-stress-responsive genes in winterrapeseed (Brassica rapa L.). Int. J. Mol. Sci. 2019; 20: 1071

33. Li Y, Wang X, Ban Q, Zhu X, Jiang C, Wei C. Comparative transcriptomic analysis reveals gene expression associated with cold adaptation in the tea plant Camellia sinensis. BMC Genomics 2019; 20: 624.

34. Gu Y, He L, Zhao C, Wang F, Yan B, Gao Y, Li Z, Yang K, Xu J. Biochemical and transcriptional regulation of membrane lipid metabolism in maize leaves under low temperature. Front Plant Sci. 2017;8:2053.

35. Zhukov A. Palmitic acid and its role in the structure and functions of plant cell membranes. Russian Journal of Plant Physiology. 2015; 62: 706-713.

36. Moellering E, Benning C. Galactoglycerolipid metabolism under stress: a time for remodeling. Trends Plant Sci. 2011;16:98-107.

37. Chen M, Thelen J. ACYL-LIPID DESATURASE2 is required for chilling and freezing tolerance in Arabidopsis. Plant Cell. 2013;25:1430-44.

38. Sakamoto A, Sulpice R, Hou C, Kinoshita M, Murata N. Genetic modification of the fatty acid unsaturation of phosphatidylglycerol in chloroplasts alters the sensitivity of tobacco plants to cold stress. Plant Cell Environ. 2004:27:99-105.

39. Gigon A, Matos A, Laffray D, Zuily-Fodil Y, Pham-Thi A. Effect of drought stress on lipid metabolism in the leaves of Arabidopsis thaliana (Ecotype Columbia). Ann Bot. 2004;94:345-51.

40. Omoto E, Iwasaki Y, Miyake H, Taniguchi M. Salinity induces membrane structure and lipid changes in maize mesophyll and bundle sheath chloroplasts. Physiol Plant. 2016;157:13-23.

41. Moellering E, Muthan B, Benning C. Freezing tolerance in plants requires lipid remodeling at the outer chloroplast membrane. Science. 2010; 330: 226-228.

42. Klaus D, Hartel H, Fitzpatrick L, Froehlich J, Hubert J, Benning C, Dormann P. Digalactosyldiacylglycerol synthesis in chloroplasts of the Arabidopsis dgd1 mutant. Plant Physiol. 2002;128:885-95.

43. Upchurch R. Fatty acid unsaturation mobilization and regulation in the response of plants to stress. Biotechnol Lett. 2008;30:967-77.
44. Yusupov R, Finegold D, Naylor E, Sahai I, Waisbren S, Levy H. Sudden death in medium chain acyl-coenzyme a dehydrogenase deficiency (MCADD) despite newborn screening. Mol Genet Metab. 2010;101:33-9.

45. Jiang T, Zhang $X$, Wang $X$, Zhang D. Arabidopsis 3-ketoacyl-CoA thiolase-2 (KAT2) an enzyme of fatty acid beta-oxidation isinvolved in ABA signal transduction. Plant Cell Physiol. 2011;52:528-38.

46. Arent S, Christensen C, Pye V, Norgaard A, Henriksen A. The multifunctional protein in peroxisomal beta-oxidation: structure and substrate specificity of the Arabidopsis thaliana protein MFP2. J Biol Chem. 2010;285:24066-77.

47. Hu Y, Jiang Y, Han X, Wang H, Pan J, Yu D. Jasmonate regulates leaf senescence and tolerance to cold stress: crosstalk with other phytohormones. J Exp Bot. 2017;68:1361-9.

48. Verma V, Ravindran P, Kumar P. Plant hormone-mediated regulation of stress responses. BMC Plant Biol. 2016;16:86.

49. Wang S, Shen G, Chen X, Zhu Q, Wang H. QTL analysis for relative water content in rice. J Agric Sci Technol. 2014;15:1849-51.

50. Song S, Chen Y, Chen J, Dai X, Zhang W. Physiological mechanisms underlying OsNAC5-dependent tolerance of rice plants to abiotic stress. Planta. 2011;234:331-45.

51. Chen N, Yang Q, Hu D, Pan L, Chi X, Chen M. Gene expression profiling and identification of resistance genes to low temperature in leaves of peanut (Arachis hypogaea L.). Sci Hortic. 2014;169:214-25.

52. Xin W, Zhang L, Zhang W, Gao J, Yi J, Zhen X, Li Z, Zhao Y, Peng C, Zhao C. An Integrated Analysis of the Rice Transcriptome and Metabolome Reveals Differential Regulation of Carbon and Nitrogen Metabolism in Response to Nitrogen Availability. Int J Mol Sci. 2019;20(9):2349.

53. Liang Y, Wang J, Zeng F, Wang Q. Chen H. Photorespiration regulates carbon-nitrogen metabolism by magnesium chelatase $\mathrm{d}$ subunit in rice. J Agricultural Food Chem. 2020; 69.

54. Li Y, Yang $X$, Ren $B$, Shen $Q$, Guo $S$. Why nitrogen use efficiency decreases under high nitrogen supply in rice (Oryza sativa L.) seedlings. J Plant Growth Regulation. 2012;31:47-52.

55. Gwak Y, Hwang Y, Wang B, Kim M, Jeong J, Lee C, Hu Q, Han D, Jin E. Comparative analyses of lipidomes and transcriptomes reveal a concerted action of multiple defensive systems against photooxidative stress in Haematococcus pluvialis. J Exp Bot. 2014;65:4317-34.

56. Vidal NP, Pham HT, Manful C, Pumphrey R, Nadeem M, Cheema M, Galagedara L, Leke-Aladekoba A, Abbey L, Thomas R. The use of natural media amendments to produce kale enhanced with functional lipids in controlled environment production system. Sci Rep. 2018;8(1):14771.

57. Xu Z, You W, Zhou Y, Chen W, Wang Y, Shan T. Cold-induced lipid dynamics and transcriptional programs in white adipose tissue. BMC Biol. 2019;17(1):74.

\section{Publisher's Note}

Springer Nature remains neutral with regard to jurisdictional claims in published maps and institutional affiliations.

$$
\begin{aligned}
& \text { Ready to submit your research? Choose BMC and benefit from: } \\
& \text { - fast, convenient online submission } \\
& \text { - thorough peer review by experienced researchers in your field } \\
& \text { - rapid publication on acceptance } \\
& \text { - support for research data, including large and complex data types } \\
& \text { - gold Open Access which fosters wider collaboration and increased citations } \\
& \text { - maximum visibility for your research: over } 100 \mathrm{M} \text { website views per year }
\end{aligned}
$$

At BMC, research is always in progress.

Learn more biomedcentral.com/submissions 